\title{
Influence of Three Types of Automated Coagulometers on the Intemational Sensitivity Index (IS) of Rabbit, Human, and Recombinant Human Tissue Factor Preparations
}

\section{A Multicenter Study}

\author{
A. M. H. P. van den Besselaar ${ }^{1}$, L. L. Houbouyan², M. F. Ailla ud ${ }^{3}$, K. W. E. Denson 4 , \\ M. J ohnston ${ }^{5}$, S. Kitc hen ${ }^{6}$, T. L. Linda hl7, M. Marren ${ }^{8}$, M. E. Martinuzzo ${ }^{9}$, C. Droullé ${ }^{10}$, \\ A. Tripodi11, C. Vergnes ${ }^{12}$
}

From the ${ }^{1}$ Haemostasis and Thrombosis Resea rch Center, Leid en University Medic al Center, Leiden, The Netherlands; ${ }^{2}$ Hôpital Ambro ise Pa ré, Service Central d'immuno-hématologie, Boulogne, France; ${ }^{3}$ Hôpital de la Timone, Laboratoire d'hématologie, Marseille, France; ${ }^{4}$ Thame Thrombosis and Haemostasis Research Foundation, Thame, Oxon, UK; ${ }^{5} \mathrm{Ha}$ milton C ivic Hosp ita Is Research Centre, Hamilton,

Ontario, Canada; ${ }^{6}$ Royal Hallamshore Hospital, Department of Haematology, Sheffield, UK; ${ }^{7}$ University Hospital, Department of C linic al Chemistry, Linköping, Sweden; ${ }^{8}$ Beaumont Hospital, Coagulation Laboratory, Department of Haematology, Dublin, Ireland; 9University Institute of Biomedic al Sciences, Fava loro Foundation, Buenos Aires, Argentina; ${ }^{10}$ Hôpital Robert Debré, Labora to ire Central d 'hématologie, Reims, France; ${ }^{11} \mathrm{~A}$. Bianc hi Bonomi Hemophilia and Thrombosis Centre, IRC C S Maggiore Hospital, Mila no, Italy; ${ }^{12}$ Hôpital Cardiologique Haut Lévêque, Laboratoire d'hémobiologie, Pessac, France

\section{Summary}

Five tissue factor reagents and three types of automated instruments for prothrombin time (PT) determination were studied in an international multicenter collaborative exercise. The purpose of this work was to determine the international sensitivity index (ISI) for each combination of reagent and instrument against the international reference preparation RBT/90. Each type of instrument was used by 3 or 4 centers to assess the interlaboratory variation of the ISI. The interlaboratory variation of the ISI for each combination of reagent and instrument ranged between $0.4 \%$ and $7.8 \%$ coefficient of variation. For three reagents, the mean ISI values for ACL (nephelometric) and STA (mechanical) were practically identical, but the mean ISI values for MLA (photo-optical) were at least 10\% higher. For two other reagents prepared from rabbit tissue, the mean ISI values increased in the order ACL, STA, MLA. The widest range of mean ISI values was noted with one rabbit tissue factor reagent: 1.68 for ACL and 2.00 for MLA. Exclusion of patient specimens with INR $<1.5$ and INR $>4.5$ determined by the international reference preparation resulted in a slight decrease of the mean ISI.

The interlaboratory variation of the International Normalized Ratio (INR) was assessed from the results obtained with common lyophilized and deep-frozen plasmas. The use of instrument-specific ISI values resulted in reduced interlaboratory variation of the INR. It is recommended that thromboplastin manufacturers provide instrumentspecific ISI values.

Correspondence to: Dr. A. M. H. P. van den Besselaar, Haemostasis and Thrombosis, Research Centre, Building 1, C2-R, Leiden University Medical Centre, P.O. Box 9600, 2300 RC Leiden, The Netherlands - Tel.: +31 71526 1894; FAX Number: +31 715266755

\section{Introduction}

The prothrombin time (scientific name: tissue factor - induced coagulation time) is widely used for monitoring oral anticoagulant treatment. The result of the test is influenced by the tissue factor (thromboplastin) reagent and the instrument used. It is recommended to report prothrombin times in terms of the international normalized ratio (INR). The INR is calculated with the formula INR $=(\mathrm{PT} / \mathrm{MNPT})^{\mathrm{ISI}}$ in which PT is the patient's prothrombin time, MNPT the mean normal prothrombin time, and ISI the international sensitivity index of the prothrombin time system used. The ISI is obtained by calibration of the system against an international reference preparation using fresh plasma samples from healthy persons and patients on long-term oral anticoagulant treatment (1). Traditionally, prothrombin times were determined with manual techniques and ISI values for thromboplastins related to the manual techniques. Today, many laboratories use automated coagulometers. Several studies have demonstrated that the ISI value is influenced by the type of instrument $(2,3)$. Some of these studies were performed by a single laboratory and their value is therefore limited. Several multicentric studies of coagulometer effects were carried out with lyophilized plasmas $(4,5)$.

The present study is the first multicentric study in which fresh plasma samples were used to assess the influence of different types of instrument on the ISI. The purpose of this study is to determine ISI values for five tissue factor reagents (two from rabbit tissue, two from human tissue, and one recombinant human) and three automated instruments. The tissue factor reagents represent materials currently used by many clinical laboratories in various countries. Two reagents were provided with instrument-specific ISI values by their respective manufacturers but the others were not.

In the present study the international reference preparation (IRP) for thromboplastin rabbit, plain (RBT/90) was used as yardstick. When this 
Table 1 Thromboplastin preparations used in this study

\begin{tabular}{|l|l}
\hline Thromboplastin & Stated ISI \\
\hline T1: RBT/90 & 1.00 (manual technique) \\
\hline T2: USP Reference Standard & 0.96 (manual technique) \\
\hline T3: Thromborel-S & 1.02 (ACL) \\
& 1.07 (bead coagulometers) \\
& 1.19 (MLA) \\
\hline T4: Hemolab ISIMAT & 0.95 (Hemolab, ACL, STA, ST4, KC10) \\
& 1.02 (MLA 1000C, Coag-a-mate X2) \\
\hline T5: Néoplastine CI & 1.82 (instrument not specified) \\
\hline T6: Néoplastine CI Plus & 1.22 (instrument not specified) \\
\hline
\end{tabular}

study was carried out, stocks of the international reference preparation for thromboplastin human, plain (BCT/253) were practically exhausted and could not be included.

Several lyophilized and deep-frozen plasma samples were provided to the participants. It could be shown that the use of instrument-specific ISI values improved the interlaboratory variation of the INR for lyophilized and frozen plasma samples.

\section{Materials and Methods}

\section{Thromboplastin Reagents}

RBT/90, the international reference preparation (IRP) for thromboplastin, rabbit, plain, was obtained from the World Health Organization (WHO) (6). The USP Thromboplastin Reference Standard, recombinant human type, was obtained from the United States Pharmacopeial Convention, Inc., Rockville, Maryland (7). Thromborel-S (lot number 505705), prepared from human placenta, was provided by the manufacturer Behringwerke AG, Marburg, Germany. Hemolab Isimat (lot number 091115 623595), prepared from human placenta, was provided by the manufacturer BioMérieux SA, Marcy-l'Etoile, France. Néoplastine CI (lot number 951193), and Néoplastine CI Plus (lot number 952186), both prepared from rabbit brain, were given by the manufacturer Diagnostica Stago, Asnières-sur-Seine, France. These six reagents are coded as T1, T2, T3, T4, T5, and T6, respectively, in the present study (Table 1). Reconstitution fluids for T2, T4, T5, and T6 were provided by the respective manufacturers.

\section{Other Reagents and Equipment}

Each participating laboratory was also provided with the following: evacuated blood collection tubes (Vacutainer, Becton Dickinson, Meylan, France), siliconized, containing $0.105 \mathrm{~mol} / \mathrm{l}$ buffered sodium citrate solution, for blood collection in the proportion of 1 volume of anticoagulant to 9 volumes of blood; sterile distilled water for reconstitution of T1, T3, and lyophilized test plasmas; sterile calcium chloride, $0.025 \mathrm{~mol} / \mathrm{l}$, for recalcification of plasma/T1 mixtures. Lyophilized pooled coumarin plasma (coded L3) was provided by Immuno AG, Vienna, Austria.

\section{Methods}

All thromboplastins were used according to the instructions of the manufacturers. The clotting times with $\mathrm{T} 1$ were determined by the manual technique (tilt tube) by all participants but one which used a Coagtester Epsilon 104 (Labover, Montpellier, France).

The clotting times with the other five thromboplastins were determined with an automated coagulometer, either STA (Diagnostica Stago), or ACL (Instrumentation Laboratory), or Electra of Medical Laboratory Automation (MLA, Pleasantville, New York). The STA is a bead coagulometer detecting a change of viscosity, the ACL is a nephelometric instrument, and the Electra is measuring light absorbance.

\section{Design of the Study}

All participants received detailed instructions on how to collect blood specimens, to handle thromboplastins and to determine prothrombin times. Calibration was carried out independently by each participant using T1 as reference system. All thromboplastins were to be tested over 10 working days. On each day a different set of fresh plasmas from 2 healthy individuals and 6 patients on oral anticoagulant treatment were used. All levels of intensity of anticoagulation were acceptable provided the patients were on oral anticoagulants for at least 6 weeks in the range of 1.5 to 4.5 INR. To minimize the effect of instability of plasma samples and reconstituted thromboplastins the testing order of thromboplastins was changed from day to day according to a form provided. Single PT determinations were performed. On each day, the same set of 13 lyophilized plasmas and 5 deep-frozen pooled plasmas were analyzed with T2, T3, T4, T5, and T6. Due to limited availability of T1, it was not possible to analyze all lyophilized and frozen plasmas with this reagent.

\section{Statistical Analysis}

ISI values were estimated for each laboratory according to the calibration protocol recommended by the WHO (1). Briefly, PTs for fresh plasmas from healthy individuals and patients were plotted on a double-logarithmic scale with $\mathrm{T} 1$ on the vertical axis and T2, T3, T4, T5, or T6 on the horizontal axis. Orthogonal regression lines were calculated for the 5 combinations of $\mathrm{T} 1$ and the other thromboplastins (8). In one analysis, all patient specimens were included irrespective of the INR determined with T1. In another analysis, specimens with INR outside the 1.5-4.5 INR interval (determined with T1) were excluded. Data points lying at a perpendicular distance from the orthogonal regression line greater than 3 standard deviations around the line were identified as outliers and were discarded for calculation of the final line. The ISI values for T2 to T6 were the product of the respective slope of the orthogonal regression line and the manual ISI for T1. Since the manual ISI for T1 is equal to 1.0 , the ISI values for the thromboplastins are equal to the values for the slope. The within-laboratory precision of the calibration line was estimated by the standard deviation (SD) of the slope of the orthogonal regression line, and was expressed as coefficient of variation (CV): $\mathrm{CV}=100 \mathrm{SD} /$ slope. It should be noted that the within-laboratory SD of the slope is not the result of repeated slope assessments by one center but was derived from the scatter of individual
Table 2 Number of normals' and patients' specimens, exclusions, and outliers. INRs of specimens were determined with RBT/90. After excluding specimens with INR $<1.5$ and those with INR $>4.5$, outliers were identified for calibration lines of $\mathrm{T} 2, \mathrm{~T} 3, \mathrm{~T} 4, \mathrm{~T} 5$, and $\mathrm{T} 6$

\begin{tabular}{|l|l|l|l|l|l|l|l|l|l|}
\hline Lab nr. & normals & patients & INR $<1.5$ & INR $>4.5$ & outliers T2 & outliers T3 & outliers T4 & outliers T5 & outliers T67 \\
\hline 1 & 20 & 60 & - & 2 & 1 & - & - & - & 1 \\
\hline 2 & 20 & 57 & 2 & 1 & 1 & 1 & - & - & - \\
\hline 3 & 20 & 60 & - & 7 & 1 & - & - & 1 & 1 \\
\hline 4 & 20 & 60 & 1 & 5 & 1 & - & - & - & 1 \\
\hline 5 & 20 & 60 & - & 5 & - & - & - & - & - \\
\hline 6 & 20 & 60 & - & 9 & 1 & 1 & 1 & 1 & 1 \\
\hline 7 & 20 & 60 & 2 & 2 & - & 1 & 1 & - & - \\
\hline 8 & 20 & 60 & - & 4 & - & - & 1 & 2 & 1 \\
\hline 9 & 20 & 60 & - & 2 & - & - & - & 1 & 1 \\
\hline 10 & 20 & 60 & - & 1 & 1 & 1 & 1 & 1 & 1 \\
\hline 12 & 20 & 60 & - & 1 & - & 1 & 1 & - & - \\
\hline
\end{tabular}


Table 3 Slopes and within-laboratory CV (in \%) of slope for orthogonal regression lines. Specimens with INR $<1.5$ or $>4.5$ were excluded for calculation of regression lines

\begin{tabular}{|l|l|l|l|l|l|l|}
\hline $\begin{array}{l}\text { Laboratory } \\
\text { number }\end{array}$ & Instrument & $\begin{array}{l}\text { Slope/CV } \\
\text { for T2 }\end{array}$ & $\begin{array}{l}\text { Slope/CV } \\
\text { for T3 }\end{array}$ & $\begin{array}{l}\text { Slope/CV } \\
\text { for T4 }\end{array}$ & $\begin{array}{l}\text { Slope/CV } \\
\text { for T5 }\end{array}$ & $\begin{array}{l}\text { Slope/CV } \\
\text { for T6 }\end{array}$ \\
\hline 3 & ACL & $0.862 / 2.9$ & $1.088 / 2.1$ & $0.990 / 2.7$ & $1.715 / 1.8$ & $1.310 / 1.9$ \\
\hline 4 & ACL & $0.896 / 2.5$ & $1.037 / 2.3$ & $0.985 / 2.4$ & $1.682 / 2.2$ & $1.312 / 2.4$ \\
\hline 8 & ACL & $0.886 / 2.1$ & $1.070 / 2.0$ & $0.993 / 2.3$ & $1.650 / 1.5$ & $1.276 / 1.7$ \\
\hline 2 & STA & $0.855 / 2.7$ & $1.071 / 2.1$ & $0.976 / 2.8$ & $1.698 / 1.4$ & $1.346 / 2.3$ \\
\hline 5 & STA & $0.895 / 2.6$ & $1.087 / 2.2$ & $1.002 / 2.6$ & $1.788 / 2.0$ & $1.370 / 2.2$ \\
\hline 9 & STA & $0.863 / 2.8$ & $1.058 / 2.3$ & $0.979 / 2.7$ & $1.861 / 2.0$ & $1.464 / 2.2$ \\
\hline 1 & MLA & $1.019 / 2.6$ & $1.132 / 2.5$ & $1.145 / 2.4$ & $1.970 / 3.0$ & $1.373 / 2.8$ \\
\hline 7 & MLA & $1.008 / 2.4$ & $1.296 / 2.8$ & $1.097 / 2.5$ & $2.075 / 2.4$ & $1.516 / 2.1$ \\
\hline 10 & MLA & $0.863 / 3.2$ & $1.183 / 3.3$ & $0.973 / 3.3$ & $1.832 / 3.2$ & $1.397 / 2.8$ \\
\hline 12 & MLA & $1.009 / 1.8$ & $1.338 / 1.7$ & $1.134 / 1.6$ & $2.105 / 1.5$ & $1.579 / 1.5$ \\
\hline
\end{tabular}

Table 4 Mean ISI and between-laboratory CV (in \%) for each instrument group, excluding plasma specimens with INR $<1.5$ or $>4.5$

\begin{tabular}{|ll|l|l|l|}
\hline Reagent & & ISI for ACL $(\mathrm{n}=3)$ & ISI for STA $(\mathrm{n}=3)$ & ISI for MLA $(\mathrm{n}=4)$ \\
\hline $\mathrm{T} 2$ & Mean: & 0.88 & 0.87 & 0.97 \\
& Range: & $0.86-0.90$ & $0.86-0.90$ & $0.86-1.02$ \\
& CV: & 2.0 & 2.4 & 7.7 \\
\hline $\mathrm{T} 3$ & Mean: & 1.07 & 1.07 & 1.24 \\
& Range: & $1.04-1.09$ & $1.06-1.09$ & $1.13-1.34$ \\
& CV: & 2.4 & 1.4 & 7.8 \\
\hline $\mathrm{T} 4$ & Mean: & 0.99 & 0.99 & 1.09 \\
& Range: & $0.98-0.99$ & $0.98-1.00$ & $0.97-1.14$ \\
& CV: & 0.4 & 1.4 & 7.3 \\
\hline T5 & Mean: & 1.68 & 1.78 & 2.00 \\
& Range: & $1.65-1.71$ & $1.70-1.86$ & $1.83-2.10$ \\
& CV: & 1.9 & 4.6 & 6.2 \\
\hline \multirow{2}{*}{6} & Mean: & 1.30 & 1.39 & 1.47 \\
& Range: & $1.28-1.31$ & $1.35-1.46$ & $1.37-1.58$ \\
& CV: & 1.6 & 4.5 & 6.7 \\
\hline
\end{tabular}

Table 5 Slopes and within-laboratory CV (in \%) of slope for orthogonal regression lines including all plasma specimens

\begin{tabular}{|l|l|l|l|l|l|l|}
\hline $\begin{array}{l}\text { Laboratory } \\
\text { number }\end{array}$ & Instrument & $\begin{array}{l}\text { Slope/CV } \\
\text { for T2 }\end{array}$ & $\begin{array}{l}\text { Slope/CV } \\
\text { for T3 }\end{array}$ & $\begin{array}{l}\text { Slope/CV } \\
\text { for T4 }\end{array}$ & $\begin{array}{l}\text { Slope/CV } \\
\text { for T5 }\end{array}$ & $\begin{array}{l}\text { Slope/CV } \\
\text { for T6 }\end{array}$ \\
\hline 3 & ACL & $0.923 / 2.8$ & $1.167 / 2.3$ & $1.069 / 2.7$ & $1.831 / 2.0$ & $1.388 / 2.0$ \\
\hline 4 & ACL & $0.937 / 2.6$ & $1.087 / 2.5$ & $1.028 / 2.6$ & $1.741 / 2.3$ & $1.374 / 2.6$ \\
\hline 8 & ACL & $0.893 / 2.0$ & $1.079 / 1.9$ & $1.005 / 2.2$ & $1.675 / 1.6$ & $1.290 / 1.7$ \\
\hline 2 & STA & $0.859 / 2.6$ & $1.075 / 2.0$ & $0.988 / 2.7$ & $1.697 / 1.4$ & $1.364 / 2.3$ \\
\hline 5 & STA & $0.917 / 2.6$ & $1.089 / 2.0$ & $1.033 / 2.5$ & $1.820 / 1.9$ & $1.409 / 2.2$ \\
\hline 9 & STA & $0.880 / 2.8$ & $1.076 / 2.3$ & $0.997 / 2.7$ & $1.891 / 2.1$ & $1.478 / 2.2$ \\
\hline 1 & MLA & $1.014 / 2.5$ & $1.141 / 2.4$ & $1.160 / 2.4$ & $1.973 / 2.9$ & $1.375 / 2.7$ \\
\hline 7 & MLA & $1.013 / 2.3$ & $1.302 / 2.5$ & $1.097 / 2.4$ & $2.045 / 2.3$ & $1.521 / 2.0$ \\
\hline 10 & MLA & $0.870 / 3.2$ & $1.169 / 3.2$ & $0.982 / 3.2$ & $1.855 / 3.2$ & $1.399 / 2.7$ \\
\hline 12 & MLA & $1.011 / 1.8$ & $1.347 / 1.8$ & $1.148 / 1.7$ & $2.113 / 1.4$ & $1.586 / 1.5$ \\
\hline
\end{tabular}

Table 6 Interlaboratory variation $(\mathrm{CV}$, in \%) of clotting times and INR for lyophilized coumarin plasma L3. INRs were calculated using local ISI based on fresh plasma testing

\begin{tabular}{|l|l|l|l|l|l|l|l|l|}
\hline \multicolumn{9}{|c|}{ CV of clotting times } \\
\hline & $\begin{array}{l}\text { ACL } \\
(\mathrm{n}=3)\end{array}$ & $\begin{array}{l}\text { STA } \\
(\mathrm{n}=3)\end{array}$ & $\begin{array}{l}\text { MLA } \\
(\mathrm{n}=4)\end{array}$ & $\begin{array}{l}\text { All } \\
(\mathrm{n}=10)\end{array}$ & $\begin{array}{l}\text { ACL } \\
(\mathrm{n}=3)\end{array}$ & $\begin{array}{l}\text { STA } \\
(\mathrm{n}=3)\end{array}$ & $\begin{array}{l}\text { MLA } \\
(\mathrm{n}=4)\end{array}$ & $\begin{array}{l}\text { All } \\
(\mathrm{n}=10)\end{array}$ \\
\hline $\mathrm{T} 2$ & 0.8 & 5.5 & 1.9 & 3.8 & 2.4 & 8.4 & 8.7 & 8.6 \\
\hline $\mathrm{T} 3$ & 3.0 & 1.5 & 4.6 & 3.9 & 2.1 & 4.6 & 7.5 & 7.6 \\
\hline $\mathrm{T} 4$ & 1.1 & 4.3 & 1.4 & 3.2 & 3.7 & 9.3 & 7.8 & 8.1 \\
\hline $\mathrm{T} 5$ & 1.5 & 2.0 & 2.0 & 2.9 & 2.3 & 4.3 & 8.3 & 7.7 \\
\hline $\mathrm{T} 6$ & 2.2 & 6.7 & 3.9 & 4.6 & 2.2 & 6.7 & 8.4 & 7.9 \\
\hline
\end{tabular}

Table 7 Mean INR and interlaboratory variation $(\mathrm{n}=10)$ for lyophilized coumarin plasma L3. INRs were calculated with the manufacturer's stated ISI and with each laboratory's own ISI determined in this study. Interlaboratory variation is expressed as coefficient of variation $(\mathrm{CV}$, in \%)

\begin{tabular}{|l|l|l|l|l}
\hline \multicolumn{3}{|c}{ Manufacturer's calibration } & \multicolumn{2}{c}{ Local calibration } \\
\hline Thromboplastin & Mean INR & CV & Mean INR & CV \\
\hline T2 & 3.34 & 8.5 & 3.15 & 8.6 \\
\hline T3 & 2.99 & 4.8 & 3.09 & 7.6 \\
\hline T4 & 3.13 & 6.2 & 3.32 & 8.1 \\
\hline T5 & 3.25 & 10.9 & 3.25 & 7.7 \\
\hline T6 & 3.01 & 11.2 & 3.49 & 7.9 \\
\hline
\end{tabular}

points about the orthogonal regression line. For each laboratory and for each reagent, INR values for the lyophilized and frozen plasmas were calculated from the mean PTs (i.e., the mean PT of 10 days' determinations), the local mean of 20 fresh normal plasma PTs, and the manufacturer's stated ISI or the local ISI determined in the present study. All calculations were performed centrally in the first author's laboratory.

\section{Results}

Eleven laboratories completed the exercise. Table 2 shows the numbers of normals and patients. Some patient specimens had INR values smaller than 1.5 or greater than 4.5 as calculated with the international reference preparation RBT/90 (T1). When these specimens were excluded for further calculations, a few outlier points were rejected because their distance from the orthogonal regression line was greater than 3 standard deviations. Table 3 shows the slopes of the orthogonal regression lines and associated standard errors for the 5 reagents obtained by each laboratory. The within-laboratory $\mathrm{CV}$ of the slopes ranged from $1.5 \%$ (laboratory nr. 12) to $3.3 \%$ (laboratory nr. 10). Since laboratory nr. 6 determined PTs with T1 using an instrument rather than the manual tilt-tube technique as required by the protocol, the results of this center were excluded.

When the laboratories were grouped according to the instrument used, the mean ISI increased in the order ACL, STA, MLA for T5 and T6 (Table 4). For T2, T3, and T4, the mean ISI values with ACL and STA were practically the same, but the mean ISI values for MLA were at least $10 \%$ greater. The between-laboratory variation for the centers using MLA was greater than the CV for the centers using ACL or STA.

Table 5 shows ISI values calculated for all data, i.e., including patient specimens with INR $<1.5$ and INR $>4.5$. The mean ISI values of Table 5 were greater than the corresponding mean values of Table 3 .

The interlaboratory variation of clotting times and INRs could be assessed from the results of lyophilized and deep-frozen plasma samples provided to the participants. The results for one lyophilized coumarin plasma are shown in Tables 6 and 7. The interlaboratory variation of the clotting times was smaller than the variation of the INRs calculated with each laboratory's own ISI as determined in this study (Table 6). INRs for this lyophilized plasma were also calculated using the manufacturers' stated ISI values (Table 7). If the manufacturer provided instrument-specific ISI values (i.e., for T3 and T4, see Table 1), the appropriate ISI was used. The stated ISI for T2 was for the manual technique only (Table 1) and this value was used for calculation of INRs in Table 7. The inter-laboratory variation of the INRs calculated with the manufacturer's instrument-specific ISI values (i. e., the values for $\mathrm{T} 3$ and $\mathrm{T} 4$ ) was smaller than the variation calculated with the stated non-specific ISI values (i. e., for T2, T5, and T6). Similar results were obtained with frozen coumarin plasma (not shown). 


\section{Disc ussion}

The purpose of the present study was to determine ISI values for 5 thromboplastin reagents used with 3 automated coagulometers and to compare these with the manufacturers' stated values. It is recommended that human thromboplastins should be calibrated with the international reference preparation (IRP) for human thromboplastin and rabbit thromboplastins with the IRP for rabbit thromboplastin (1). This is because the precision of calibration is improved when similar thromboplastins are being compared. In the present study both human and rabbit thromboplastins were calibrated with the IRP for rabbit thromboplastin. Even though this may have resulted in suboptimal precision for the human thromboplastins, the mean within-laboratory $\mathrm{CVs}$ of the calibration slopes were not greater than the $3 \%$ limit imposed by the WHO requirements (Table 3 ). The relative effect of different instruments on the ISI of the human and rabbit thromboplastins is not influenced by the IRP, because all participants used the same IRP and the same (manual) technique for the IRP. We felt that the use of a single IRP was justified for the purpose of the present study.

The coagulometers were based on different end point detection principles: nephelometric (ACL), viscometric (STA), and measurement of light absorbance (MLA). There was good agreement between the three laboratories using ACL instruments (Tables 3 and 4). Among the four laboratories using MLA instruments, there was one (nr. 10) which had $>10 \%$ smaller ISI for T2 and T4 than the other three laboratories. The cause of this difference is not known.

The between-laboratory CV of the ISI for the MLA laboratories was considerably greater than the within-laboratory $\mathrm{CV}$, suggesting that there were systematic differences between the laboratories. Part of the systematic differences may be caused by small local modifications of the manual technique for the international reference preparation RBT/90 (T1), and/or technical differences between the instruments. The magnitude of the ISI differences between the individual laboratories was not the same for all reagents (Table 3), suggesting that local modification of the method used for T1 was not the only cause.

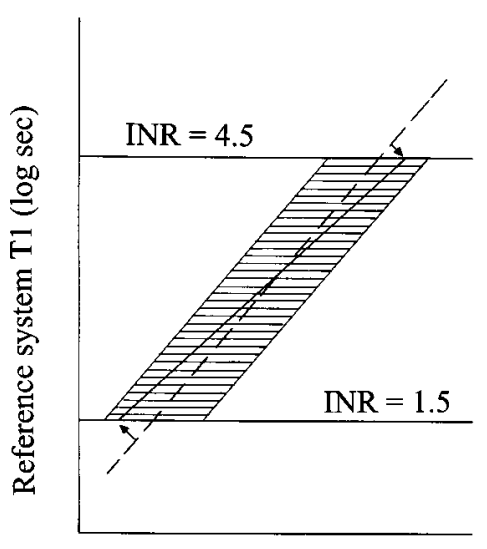

Test system $(\log s e c)$

Fig. 1 Effect of selection of patient specimens on calculation of calibration line. Log prothrombin times determined with the reference system (in this case T1) are plotted on the vertical axis; log prothrombin times with the test system (either T2, T3, T4, T5, or T6) on the horizontal axis. The dashed line represents the true line of relationship. The shaded area represents the scatter of individual patient data obtained after data points were selected by using cut-off lines parallel to the horizontal axis corresponding to INR values of 1.5 and 4.5 respectively
There was a trend of greater ISI values for the laboratories using MLA instruments than those obtained by the three laboratories using ACL instruments. This trend was observed for all five reagents. Our results support the notion that it is not possible to assign a single ISI value to a reagent which is valid for all coagulometer systems. Two reagents (T3 and T4) were supplied with instrument-specific ISI values by their respective manufacturers. The ISI values provided by the manufacturer for T3 and various instruments, were 0-5\% smaller than the mean values obtained in the present study (cf. Tables 1 and 4). Likewise, the ISI values provided by the manufacturer for T4 were 4-7\% smaller than the corresponding mean values of the present study. The manufacturer of T6 supplied a single ISI value (1.22) which was considerably smaller than all values determined in the present study. The manufacturer informed us that the stated ISI had been determined using samples collected with $0.129 \mathrm{~mol} / \mathrm{l}$ sodium citrate. The participants of the present study used $0.105 \mathrm{~mol} / \mathrm{l}$ sodium citrate. It is possible that the lower ISI value stated by the manufacturer is the result of using a higher sodium citrate concentration $(9,10)$.

The ISI values for T2 determined in the present study are slightly different from the mean value reported for the manual technique, i.e. 0.96 (7). Apart from a different technique of clotting time determination, the use of different reference preparations may account for minor ISI differences (11). RBT/90 was used in the present study, but BCT/441 was used by the manufacturers of T2, T4, and T6. According to WHO guidelines, rabbit thromboplastins should be calibrated with RBT/90, and human thromboplastins with BCT/253. BCT/253 stocks were practically exhausted when the present study was carried out (11). However, recent multicenter studies have shown that mean ISI values obtained with RBT/90 were very similar to those obtained with BCT/253 (12).

Selection of patient samples within the range 1.5-4.5 INR is required because the calibration relation should be valid for this range. Patient samples with INR $<1.5$ or $>4.5$ are likely obtained from patients in the induction phase of treatment or from other non-stabilized patients, i.e. samples with discrepant levels of coagulation factors VII, X, and II. Non-stabilized samples are associated with greater scatter of points in a calibration plot and greater imprecision of the calibration line (13).

ISI values were slightly influenced by selection of the patient specimens for the calibration. When all specimens were used, ISI values were on average $2.4 \%$ greater than the values calculated by excluding INRs smaller than 1.5 and greater than 4.5 (cf. Tables 3 and 5). The excluded INRs were calculated with T1, that is the thromboplastin which was used as reference system for calculation of the slope and ISI. A simple geometrical consideration can account for the systematic decrease of the slope induced by excluding values in the vertical direction only, as shown in Fig. 1 (14). As a result of this method of patient specimen selection, the data points are not distributed symmetrically about the true calibration line. The slope of the orthogonal regression line calculated for the selected specimens is smaller than the slope of the true line. The magnitude of the slope bias is related to the scatter of points around the line: the greater the scatter, the greater the bias. The bias may be avoided by using a third system for selection of samples. We recommend that patient samples for calibration should be selected by using an independent prothrombin time system. Since the specimens are usually provided by an anticoagulant clinic, the system used by the clinic for routine monitoring of the patients could be used to select the specimens that fit the 1.5-4.5 INR interval suggested by the WHO guidelines. Once selected, the specimens should be used for the calibration even if the INRs determined with the reference system appear to be outside the 1.5-4.5 INR range. 
The lyophilized plasmas included in this study could be used to assess the interlaboratory variation of the clotting times (Table 6). The differences between the three laboratories using ACL instruments were smaller than the differences between the participants using STA or MLA. The interlaboratory variation of the corresponding INRs was greater (Table 6), but the differences between the three ACLs were again smaller than those between the three STAs or between the four MLAs. It is no surprise that the interlaboratory variation of the INRs is greater than that of the clotting times, because the error in the INR is a result of error in both the clotting times and the ISI values. The interlaboratory variation of the INRs in this study compared well to that in previously published reports (15-17).

The interlaboratory variation of the INR of lyophilized coumarin plasma was lower with instrument-specific ISI values than with a single ISI for all instruments (Table 7). This finding is to be expected when instruments have a significant influence on the ISI. In fact, the interlaboratory variation of the INRs calculated with the instrument-specific ISI values stated by the manufacturers of T3 and T4 was even lower than the CVs with the local ISI values (Table 7). These results suggest that the stated ISI values for T3 and T4 were accurate, and that the instruments used in the present study were very similar to those used by the manufacturers. It is recommended that thromboplastin manufacturers provide instrument-specific ISI values for their products.

\section{Acknowledgements}

We thank the staff of the participant laboratories. RBT/90 and the USP Thromboplastin Human Recombinant Reference Standard were provided by the World Health Organization (custodian: Central Laboratory of the Netherlands Red Cross Blood Transfusion Service) and the United States Pharmacopeial Convention, Inc., respectively. Commercial thromboplastins were given by Behringwerke, BioMérieux, and Diagnostica Stago. Financial support was obtained from Immuno AG, Behringwerke AG, Ortho Diagnostic Systems, and Instrumentation Laboratory Company.

\section{References}

1. WHO Expert Committee on Biological Standardization. Thirtythird Report. WHO Tech Rep Ser 1983; 687: 81-105.

2. Poggio M, Van den Besselaar AMHP, Van der Velde EA, Bertina RM. The effect of some instruments for prothrombin time testing on the International Sensitivity Index (ISI) of two rabbit tissue thromboplastin reagents. Thromb Haemost 1989; 62: 868-74.

3. Ray MJ, Smith IR. The dependence of the International Sensitivity Index on the coagulometer used to perform the prothrombin time. Thromb Haemost 1990; 63: 424-9.

4. Clarke K, Taberner DA, Thomson JM, Morris JA, Poller L. Assessment of value of calibrated lyophilized plasmas to determine International Sensitivity Index for coagulometers. J Clin Pathol 1992; 45: 58-60.
5. Poller L, Triplett DA, Hirsh J, Carroll J, Clarke K. The value of plasma calibrants in correcting coagulometer effects on International Normalized Ratios. Am J Clin Pathol 1995; 103: 358-65.

6. Van den Besselaar. Multi-center study of replacement of the international reference preparation for thromboplastin, rabbit, plain. Thromb Haemost 1993; 70: 794-9.

7. Poller L, Houghton D, Outschoorn AS. Statistical analysis of results of a collaborative study of a human recombinant tissue thromboplastin preparation to serve as a USP reference standard (collaborative assay by comparative clotting times). Pharmacopeial Forum 1996; 22: 2946-55.

8. Van der Velde EA. Orthogonal regression equation. In: Thromboplastin Calibration and Oral Anticoagulant Control. Van den Besselaar AMHP, Gralnick HR, Lewis SM, eds. Boston: Martinus Nijhoff Publishers 1984: 25-39.

9. Duncan EM, Casey CR, Duncan BM, Lloyd JV. Effect of concentration of trisodium citrate anticoagulant on calculation of the International Normalized Ratio and the International Sensitivity Index of Thromboplastin. Thromb Haemost 1994: 72: 84-8.

10. Chantarangkul V, Tripodi A, Clerici M, Negri B, Mannucci PM. Assessment of the influence of citrate concentration on the international normalized ratio (INR) determined with twelve reagent-instrument combinations. Thromb Haemost 1998; 80: 258-62.

11. Tripodi A, Poller L, Van den Besselaar AMHP, Mannucci PM. A proposed scheme for calibration of international reference preparations of thromboplastin for the prothrombin time. Thromb Haemost 1995; 74: 1368-9.

12. Tripodi A, Chantarangkul V, Negri B, Clerici M, Mannucci PM. International Collaborative Study for the Calibration of a Proposed Reference Preparation for Thromboplastin, Human Recombinant, Plain. Thromb Haemost 1998; 79: 439-43.

13. Tomenson JA. A statistician's independent evaluation. In: Thromboplastin Calibration and Oral Anticoagulant Control. Van den Besselaar AMHP, Gralnick HR, Lewis SM, eds. Boston: Martinus Nijhoff Publishers 1984: $87-108$.

14. Van den Besselaar AMHP, Van der Velde EA. The manufacturers' study. In: Thromboplastin Calibration and Oral Anticoagulant Control. Van den Besselaar AMHP, Gralnick HR, Lewis SM, eds. Boston: Martinus Nijhoff Publishers 1984: 127-49.

15. Brien WF, Crawford L, Wood DE. Discrepant Results in INR testing. Thromb Haemost 1994; 72: 986-7.

16. Kitchen S, Jennings I, Woods TAL, Walker ID, Preston FE. Two recombinant tissue factor reagents compared to conventional thromboplastins for determination of International Normalised Ratio: a thirty-three-laboratory collaborative study. Thromb Haemost 1996; 76: 372-6.

17. Houbouyan LL, Goguel AF. Long-term French experience in INR standardization by a procedure using plasma calibrants. Am J Clin Pathol 1997; 108: 83-9.

Received February 27, 1998 Accepted after resubmission September 23, 1998 